\title{
Influence of doping and solid solution formation on the thermoelectric properties of chalcopyrite semiconductors
}

\author{
Winston D. Carr ${ }^{1}$ and Donald T. Morelli ${ }^{1,2}$ \\ ${ }^{1}$ Department of Physics \& Astronomy, Michigan State University, East Lansing, MI 48824 \\ ${ }^{2}$ Department of Chemical Engineering \& Materials Science, Michigan State University, East
}

Lansing, MI 48824

\begin{abstract}
We have investigated the influence of zinc doping on the thermoelectric properties of $\mathrm{CuInTe}_{2}-\mathrm{CuGaTe}_{2}$ solid solution alloys. Undoped end-member compounds display typical p-type semiconducting behavior, with a negative temperature coefficient of resistivity and large Seebeck coefficient. With zinc substituting for indium or gallium, the hole concentration is increased and the electrical transport behavior evolves into that of a degenerate semiconductor, with both electrical resistivity and Seebeck coefficient increasing with temperature up to the highest temperature measured. For undoped samples the thermoelectric power factor is maximized close to $750 \mathrm{~K}$, while in doped specimens the maximum occurs at much lower temperature. Substitution of gallium for indium induces significant phonon scattering and thermal conductivity reduction below $500 \mathrm{~K}$. The dimensionless figure of merit rises to above unity over a range of compositions in these chalcopyrite compounds, with optimized samples reaching a figure of merit of 1.3 .
\end{abstract}

Keywords: Semiconductors; Powder metallurgy; Thermoelectric 


\section{Introduction}

Thermoelectric power generation, with its ability to convert heat directly into electricity, offers the promise of enhanced efficiency for power generation at both large and small scales, from automobiles and power plants, to interstellar space propulsion. However, low device performance and high material cost have relegated it largely to niche applications. The performance of a thermoelectric material is typically characterized by $z T$, the dimensionless figure of merit, which is given by $z T=S^{2} \sigma T \kappa^{-1}$, where $S$ is the Seebeck coefficient, $\sigma$ is electrical conductivity, $\kappa$ is thermal conductivity and $T$ is temperature. Traditionally, thermoelectric materials have possessed $z T$ values on the order of or less than unity, limiting device conversion efficiency to significantly less than $10 \%$.

In the last two decades, however, there has been a significant resurgence in thermoelectric materials research as new concepts, synthesis strategies, and understanding have steadily pushed $z T$ values ever higher. In particular, materials based on traditional $\mathrm{Pb}$ chalcogenide semiconductors, but modified to include nanostructured second phases, have undergone significant recent development. By taking advantage of a number of new approaches, including the combination of defects across length scales to scatter a wide range of phonons [1], "endotaxial" nanostructuring [2], band convergence and band engineering [3-7], anharmonicity [8-10], and resonant bonding [11] the dimensionless figure of merit has been raised to values in excess of 2.5 at high temperatures. At the same time, workers have realized that the low abundance of some elements, such as Te, and the toxicity of others, such as $\mathrm{Pb}$, may place a limitation on the application of thermoelectric materials containing these elements. Recent work has sought enhanced thermoelectric performance on inexpensive and earth-abundant materials 
such as $\mathrm{Mg}_{2} \mathrm{Si}$-based compounds [12-15], organic conductors [16], inorganic-organic perovskites [17] and even materials based on natural minerals such as tetrahedrite [18-21] and earthabundant copper sulfide [22], which offer quite good thermoelectric performance at a potentially greatly reduced cost.

The success in finding good thermoelectric performance in compounds based on natural minerals has led us to consider other potential mineral families that may provide such behavior. While tetrahedrites, of nominal composition $\mathrm{Cu}_{12-\mathrm{x}} \mathrm{Zn}_{x} \mathrm{Sb}_{4} \mathrm{~S}_{13}$, are the most abundant sulfosalts in the world and a minor ore of copper, the most common copper mineral is chalcopyrite, $\mathrm{CuFeS}_{2}$. This particular compound is just one of many isostructural compounds known generally as chalcopyrites, many of which have undergone development as photovoltaic materials. Given the wide range of compositions available in this family, and the corresponding wide range of energy band gaps, their potential application as thermoelectric materials deserves some scrutiny, and even the question of whether natural mineral chalcopyrite itself may, as in the case of tetrahedrite, serve as a source material for low-cost thermoelectrics. Indeed, Mori, et al. recently reported upon their investigations of the thermoelectric properties of $\mathrm{CuFeS}_{2}$ [23], while others have explored the influence of lattice defects [24] and nanostructure [25] on thermoelectricity in chalcopyrites.

As in the case of tetrahedrites, before an investigation of the natural mineral itself as source thermoelectric material can be successfully carried out, it is desirable to study the thermoelectric properties of samples produced under ideal conditions in the laboratory, in order to assess the range of capability with respect to thermoelectric behavior of this family of compounds. In fact, some experimental studies in this area have already been reported. Recent work on tellurium-based chalcopyrite materials, for instance, has shown much promise, with 
reported $z T$ values in excess of unity for both $\mathrm{CuInTe}_{2}$ [26] and $\mathrm{CuGaTe}_{2}$ [27]. These very promising values for the figure of merit were obtained in the nominally pure ternary compounds without the use of doping to optimize the electronic properties or of solid solutions to reduce the lattice thermal conductivity. In the present work, therefore, we have undertaken an investigation of the synthesis and thermoelectric properties of $\mathrm{Cu}\left[\operatorname{In}_{1-\mathrm{x}} \mathrm{Ga}_{\mathrm{x}}\right]_{1-\mathrm{y}} \mathrm{Zn}_{\mathrm{y}} \mathrm{Te}_{2}(0 \leq \mathrm{x} \leq 1 ; 0 \leq \mathrm{y} \leq 0.15)$. Nominally divalent $\mathrm{Zn}$ could act as either a donor on the nominally monovalent $\mathrm{Cu}$ site or as an acceptor on the nominally trivalent $[\mathrm{In}, \mathrm{Ga}]$. Here, we adjust the composition to provide for the latter case, and we show that indeed $\mathrm{Zn}$ is a p-type dopant in these compounds with capability of modifying the electrical properties. Meanwhile, we also explore the formation of solid solutions by substitution of In for Ga in order to reduce the lattice thermal conductivity of the material. These two effects act synergistically to increase $z T$ of optimized composition to values close to 1.3 at $850 \mathrm{~K}$.

\section{Materials and methods}

Samples were synthesized by reacting raw starting elements (Alfa Aesar): $\mathrm{Cu}$, In, Ga (99.999\% each) and Zn, Te (99.9999\%). Materials were weighed in stoichiometric amounts and placed in quartz ampoules, which were subsequently evacuated to less than $10^{-5}$ Torr and sealed with a methane-oxygen torch. Sealed ampoules were then suspended in a vertical tube furnace

and heated at a rate of $1^{\circ} \mathrm{C} \min ^{-1}$ to a temperature of $900^{\circ} \mathrm{C}$, where they were soaked for 24 hours. Samples were then cooled at a rate of $1^{\circ} \mathrm{C} \min ^{-1}$ to $600^{\circ} \mathrm{C}$, and held for another 24 hours, after which they were quenched in water. The resulting ingots were then placed in an argon filled stainless steel SPEX vial and ball-milled for one hour on a SPEX Sample Prep Mixer/Mill 
$8000 \mathrm{M}$. The resultant powder was hot-pressed in an argon atmosphere at $90 \mathrm{MPa}$ and $475^{\circ} \mathrm{C}$ for 15 minutes. Although the ingots were fragile and contained cracks, the hot pressed specimens were high density (92\% using the Archimedes method) and mechanically robust, making them suitable for transport measurements.

X-ray diffraction was performed on a Rigaku Miniflex II at room temperature, and patterns were indexed using the Jade 9 software package and database. From $80 \mathrm{~K}$ to $350 \mathrm{~K}$ the Seebeck coefficient, electrical resistivity, and thermal conductivity were measured with a standard 4-probe steady-state technique in a liquid nitrogen flow cryostat; details of this measurement setup can be found in a previous publication [28]. From 350 to $900 \mathrm{~K}$ the Seebeck coefficient and electrical resistivity measurements were performed on an Ulvac ZEM-3 system in an argon atmosphere. Thermal diffusivity measurements in this temperature range were obtained on a Netzsch LFA 457, and were combined with the measured densities and specific heat (Dulong-Petit values) to yield thermal conductivity values. Hall measurements were performed from 80 to 300 Kelvin, with a field strength ranging over \pm 1.5 Tesla. Carrier concentration was determined using a single band model and assuming a Hall factor of unity. We estimate an uncertainty in resistivity, Hall, and Seebeck coefficient of $5 \%$ and that in thermal conductivity of $10 \%$. The use of two different techniques at low and high temperature induces a slight discontinuity in the data at $350 \mathrm{~K}$ that is within the measurement uncertainty.

\section{Results and discussion}

\subsection{XRD and lattice parameters}


The x-ray diffraction patterns (Fig. 1) for all samples exhibit only a single phase indexing to the chalcopyrite crystal structure. We find that the two lattice parameters $a$ and $c$ characterizing this crystal structure display a linear shift with composition, as expected from Vegard's law. We do observe a fluctuation in the ratio $c / a$ from 1.99 to 1.97 in moving from $\mathrm{CuInTe}_{2}$ to $\mathrm{CuGaTe}_{2}$, consistent with the published values of 2.00 [26] and 1.98 [27]. The deviation of the $c / a$ from a value of 2.0 is due to a slight distortion of the tetrahedral arrangement around the Te atom due to the two different bond lengths ( $\mathrm{Cu}-\mathrm{Te}$ and $\mathrm{In} / \mathrm{Ga}-\mathrm{Te})$. The observed XRD patterns match very well with published studies on their structure, and we conclude that our samples are all single phase chalcopyrite with little or no presence of impurity phases [29].

\subsection{Electrical properties}

We first studied the influence of $\mathrm{Zn}$ doping on the pure end member compound $\mathrm{CuInTe} 2$. As expected, with increasing Zn we observe a decrease in electrical resistivity (Fig. 2a), as well as a decrease in Seebeck coefficient (Fig. 2b). The thermoelectric power factor $S^{2} \sigma$ was found to be optimized at a doping level of $1 \%$. The addition of $1 \%$ zinc on the indium atomic site resulted in an increase in room temperature hole concentration from $5.6 \times 10^{17} \mathrm{~cm}^{-3}$ to $3.4 \times 10^{19} \mathrm{~cm}^{-3}$ for $\mathrm{CuInTe}_{2}$, while for $\mathrm{CuGaTe}_{2}$ an increase of $1.4 \times 10^{18}$ to $6.2 \times 10^{19} \mathrm{~cm}^{-3}$ was found. Along with the increase in carrier concentration, we see a decrease in electrical resistivity of up to two orders of magnitude at room temperature, and a change from a typical semiconductor temperature dependence, to an increasing electrical resistivity as a function of temperature, characteristic of that of a degenerately doped semiconductor. These factors confirm that the zinc is primarily located on the indium lattice site and is acting as a p-type dopant. 
Subsequent to this doping study on $\mathrm{CuInTe}_{2}$, we investigated the influence of $\mathrm{Ga}$ substitution for In in both undoped samples and samples doped at the optimum level of $1 \% \mathrm{Zn}$. For the undoped series, although the magnitudes differ from sample to sample, we find that the resistivity (Fig. 3a) maintains a semiconducting nature throughout the solid solution, while the Seebeck coefficient (Fig. 3b) peaks near approximately 400K for all samples in the solidsolution. Due to the similar band gaps of the end components, we would expect this behavior [26,27]. For the undoped samples, the power factor peaks (Fig. 3c) near 750K, with $\mathrm{CuGaTe}_{2}$ having the highest value at $12 \mu \mathrm{Wcm}^{-1} \mathrm{~K}^{-2}$, and decreases with increasing amount of indium, to $8 \mu \mathrm{Wcm}^{-1} \mathrm{~K}^{-2}$ for $\mathrm{CuInTe}_{2}$. While the values for electrical resistivity and Seebeck coefficient vary from those published, the resulting power factor is in agreement. This is expected, as Liu et al. [26] showed that annealing time can vary the electrical properties, but ultimately not the zT of the sample [26,27]. For the samples doped with zinc, the resistivity (Fig. 4a) and Seebeck coefficient (Fig. 4b) again reflect those of a degenerately doped semiconductor, with both decreasing with increasing Ga concentration. However, unlike the undoped samples, the zinc doped samples show a Seebeck coefficient which increases monotonically with temperature; no peak in Seebeck coefficient is seen for the doped samples over the temperature ranges measured, and as a result it approaches the values of the undoped samples at temperatures above $750 \mathrm{~K}$. In the zinc doped series, the peak in power factor (Fig. 4c) shifts to lower temperatures, with $\mathrm{CuIn}_{.99} \mathrm{Zn}_{.01} \mathrm{Te}_{2}$ exhibiting a maximum power factor at $515 \mathrm{~K}$ with a value of $11.7 \mu \mathrm{Wcm}^{-1} \mathrm{~K}^{-2}$ and $\mathrm{CuGa}_{99} \mathrm{Zn}_{.01} \mathrm{Te}_{2}$ peaking at $712 \mathrm{~K}$, with a value of $12.5 \mu \mathrm{Wcm}^{-1} \mathrm{~K}^{-2}$. The net effect of doping by $\mathrm{Zn}$ is to yield a large increase in power factor from room temperature up to around $700 \mathrm{~K}$, at which point the power factors for the two series, doped and undoped, begin to overlap. Thus it is clear that by varying the $\mathrm{Zn}$ concentration over the range of zero to one percent, the power factor 
may be maintained at a level near $12.5 \mu \mathrm{Wcm}^{-1} \mathrm{~K}^{-2}$ over the temperature range of 400 to $800 \mathrm{~K}$.

\subsection{Thermal properties}

Below $350 \mathrm{~K}$ thermal conductivity measurements are performed along the direction of hot-pressing, using a 4 probe steady-state technique. Above this temperature, thermal diffusivity is measured with laser-flash technique, and combined with the room temperature density and Dulong-Petit value of specific heat. The laser-flash method is measuring thermal conductivity perpendicular to the pressing direction, however given the values match across the temperature ranges within error bars, we conclude that the samples are isotropic and direction is not a factor. As zinc is added to pure $\mathrm{CuInTe}_{2}$, we expect an increase in the electronic thermal conductivity due to the strongly reduced electrical resistivity in the doped samples. The Wiedemann-Franz law was used to estimate this electronic contribution (using the free electron value of the Lorenz number, which is justified for the high levels of doping in our samples [30]. We find that even in the highest doped samples $(\mathrm{y}=0.15)$, the lattice portion of thermal conductivity constitutes nearly $90 \%$ of the total thermal conductivity, and for $y=0.01$, the electronic portion is only $4 \%$ of the total thermal conductivity. Due to the low concentration of zinc used as dopant, we expect, and observe, no effect on the lattice portion of thermal conductivity.

For both doped and undoped samples (Figs. 5a and 5b), the thermal conductivity is higher for $\mathrm{CuGaTe}_{2}$ than for $\mathrm{CuInTe}_{2}$, as is expected due to the higher mass of indium; all members of the solid solution have lower values. However, while at low temperatures (80-300K) the reduction is as much as $50 \%$, above $500 \mathrm{~K}$, Umklapp scattering dominates, and all the samples

trend toward the same range of values, reaching a minimum of approximately $0.7 \mathrm{~W} \mathrm{~m}^{-1} \mathrm{~K}^{-1}$ for 
both $\mathrm{CuIn}_{0.25} \mathrm{Ga}_{0.75} \mathrm{Te}_{2}$ and $\mathrm{CuIn}_{0.99} \mathrm{Zn}_{0.01} \mathrm{Te}_{2}$ near $850 \mathrm{~K}$. We conclude that up to approximately $500 \mathrm{~K}$, forming a solid solution of $\mathrm{CuInTe}_{2}$ and $\mathrm{CuGaTe}_{2}$ can provide some reduction in thermal conductivity, however above this temperature, intrinsic phonon scattering processes dominate in all compositions, negating any benefit from additional point defect scattering.

\subsection{Thermoelectric figure of merit}

Combining the results for Seebeck coefficient, electrical resistivity, and thermal conductivity, we determine the dimensionless figure of merit for our samples; the results are displayed in Fig. 5c. The highest $z T$ for any sample is approximately 1.3 at $865 \mathrm{~K}$ for $\mathrm{Cu}\left(\mathrm{In}_{0.25} \mathrm{Ga}_{0.75}\right)_{0.99} \mathrm{Zn}_{0.01} \mathrm{Te}_{2}$, with several other samples possessing $z T$ in excess of unity. We observe a general trend of decreasing $z T$ with increasing indium content. The reason for this behavior is that at the highest temperatures there is no benefit to thermal conductivity from the solid solution, and the gallium samples have higher power factors. This, however, is not the case in the intermediate temperature range $(400 \mathrm{~K}$ to $700 \mathrm{~K})$ where a clear benefit is obtained from indium substitution, with $\mathrm{Cu}\left[\operatorname{In}_{0.65} \mathrm{Ga}_{0.35}\right]_{0.99} \mathrm{Zn}_{0.01} \mathrm{Te}_{2}$ having the highest $z T$ in the range.

\section{Conclusions}

We have studied the thermoelectric properties of compounds based on the chalcopyrite structure semiconductors $\mathrm{CuGaTe}_{2}$ and $\mathrm{CuInTe}_{2}$. Substitution of $\mathrm{Zn}$ on the group III cation site can effectively dope the material p-type and can be used to optimize the thermoelectric power factor. Solid solutions of the end member compounds induce a reduction of lattice thermal 
conductivity up to at least $500 \mathrm{~K}$. Through these compositional modifications the dimensionless figure of merit can reach values as high as 1.3 , rivalling the best p-type thermoelectric semiconductors for power generation. Our work shows that the chalcopyrite semiconductor family can offer new prospects for high performance thermoelectrics. Several compounds in this family have band gaps suitable for use in this application, and there is an opportunity to explore compounds synthesized from earth-abundant elements, and even to consider, as is the case for tetrahedrite, using natural chalcopyrite-based minerals as source thermoelectric materials.

\section{Acknowledgments}

This work was supported as part of the Revolutionary Materials for Solid State Energy Conversion, an Energy Frontier Research Center funded by the U.S. Department of Energy, Office of Science, and Office of Basic Energy Sciences under Award Number DE-SC0001054. 


\section{References}

[1] K. Biswas, J.Q. He, I. D. Blum, C.-I Wu, T. P. Hogan, D. N. Seidman, V. P. Dravid, M. G. Kanatzidis, Nature 489 (2012) 414-418.

[2] L.-D. Zhao, J.Q. He, S. Hao, C.-I Wu, T. P. Hogan, C. Wolverton, V. P. Dravid, M.G. Kanatzidis, J. Am. Chem. Soc. 134 (2012) 16327-16336.

[3] Y. Pei, X. Shi, A. LaLonde, H. Wang, L. Chen, G. J. Snyder, Nature 473 (2011) 66-69.

[4] W. Liu, X. Tan, K. Yin, H. Liu, X. Tang, J. Shi, Q. Zhang, C. Uher, Phys. Rev. Lett. 108 (2012) 166601.

[5] H. J. Wu, L.-D. Zhao, F. S. Zheng, D. Wu, Y. L. Pei, X. Tong, M. G. Kanatzidis, J. Q. He, Nat. Commun. 5 (2014) 4515.

[6] Y. Lee, S.-H. Lo, C. Chen, H. Sun, D.-Y. Chung, T.C. Chasapis, C. Uher, V. P. Dravid, Mercouri G. Kanatzidis, Nat. Commun. 5 (2014) 3640.

[7] H. Wang, Z. M. Gibbs, Y. Takagiwa, G. J. Snyder, Energy Environ. Sci. 7 (2014) 804811.

[8] E.J. Skoug and D.T. Morelli, Phys. Rev. Lett. 107 (2011) 235901.

[9] M.D. Nielsen, V. Ozolins, J.P. Heremans, Energy Environ. Sci. 6 (2012) 570-578.

[10] L.-D. Zhao, S.-H. Lo, Y. Zhang, H. Sun, G. Tan, C. Uher, C. Wolverton, V.P. Dravid, M.G. Kanatzidis, Nature 508 (2014) 373-377.

[11] S. Lee, K. Esfarjani, T. Luo, J. Zhou, Z. Tian, G. Chen, Nat. Commun. 5 (2014) 3525

[12] G.S. Nolas, D. Wang, M. Beekman, Phys. Rev. B 76 (2007) 235204

[13] M. Akasaka,T. Iida, A. Matsumoto, K. Yamanaka, Y. Takanashi, T. Imai, N. Hamada, J. Appl. Phys. 104 (2008) 013703.

[14] X. Han, G. Shao, J. Appl. Phys. 112 (2012) 013715. 
[15] G. Jiang, J. He, T. Zhu, C. Fu, X. Liu, L. Hu, X. Zhao, Adv. Funct. Mater. 24 (2014) 3776-3781.

[16] Q. Zhang, Y. Sun, W. Xu, D. Zhu, Adv. Mater. 26 (2014) 6829-6851.

[17] Y. He and G. Galli, Chem. Mater. 26 (2014) 5394-5400.

[18] X. Lu, D.T. Morelli, Y. Xia, F. Zhou, V. Ozolins, H. Chi , X. Zhou, C. Uher, Adv. Energy Mater. 3 (2012) 342-348.

[19] X. Lu, D.T. Morelli, Phys. Chem. Chem. Phys. 15 (2013) 5762-5766.

[20] X. Lu, D.T. Morelli, MRS Commun. 3 (2013) 129-133.

[21] J. Heo, G. Laurita, S. Muir, M.A. Subramanian, D.A. Keszler, Chem. Mater. 26 (2014) 2047-2051.

[22] Y. He, T. Day, T. Zhang, H. Liu, X. Shi, L. Chen, G.J. Snyder Adv. Mater. 26 (2014) 3974-3978.

[23] N. Tsujii, T. Mori, Y. Isoda, J. Electron. Mater. 43 (2014) 2371-2375.

[24] J. Yang, S. Chen, Z. Du, X. Liu, J. Cui, Dalton Trans. 43 (2014) 15228-15236.

[25] A. Kosuga, K. Umekage, M. Matsuzawa, Y. Sakamoto, I. Yamada, Inorg. Chem. 53 (2014) 6844-6849.

[26] R. Liu, L. Xi, H Liu, X. Shi, W. Zhang, L. Chen, Chem. Commun. 48 (2012) 3818-3820.

[27] T. Plirdpring, K. Kurosaki, A. Kosuga, T. Day, S. Firdosy, V. Ravi, G.J. Snyder, A. Harnwunggmoung, T. Sugahara, Y. Ohishi, H. Muta, S. Yamanaka, Adv. Mater. 24 (2012) $3622-3626$.

[28] G.J. Lehr, D.T. Morelli, Intermetallics 32 (2013) 225-229.

[29] M. Leon, J. M. Merino, J. L. Martin De Vidales, J. Vac. Sci. Technol. A11 (1993) 24302436.

[30] H. J. Goldsmid, Applications of Thermoelectricity, Methuen, London, 1960. 


\section{Figure captions}

Fig. 1 X-ray powder diffraction patterns for $\mathrm{CuIn}_{1-\mathrm{x}} \mathrm{Ga}_{\mathrm{x}} \mathrm{Te}_{2}$ and doped end members of the solid solution.

Fig. 2 Electrical resistivity (a) and Seebeck coefficient (b) of $\mathrm{CuIn}_{1-\mathrm{y}} \mathrm{Zn}_{\mathrm{y}} \mathrm{Te}_{2}$.

Fig. 3 Electrical transport properties of nominally undoped $\mathrm{CuIn}_{1-\mathrm{x}} \mathrm{Ga}_{\mathrm{x}} \mathrm{Te}_{2}$ : a) electrical resistivity; b) Seebeck coefficient; and c) thermoelectric power factor.

Fig. 4 Electrical transport properties of $\mathrm{CuIn}_{1-\mathrm{x}} \mathrm{Ga}_{\mathrm{x}} \mathrm{Te}_{2}$ doped with $1 \% \mathrm{Zn}$ on the (In,Ga) site: a) electrical resistivity; b) Seebeck coefficient; and c) thermoelectric power factor.

Fig. 5 Thermal conductivity of a) undoped $\mathrm{CuIn}_{1-\mathrm{x}} \mathrm{Ga}_{\mathrm{x}} \mathrm{Te}_{2}$ and b) $\mathrm{CuIn}_{1-\mathrm{x}} \mathrm{Ga}_{\mathrm{x}} \mathrm{Te}_{2}$ doped with $1 \% \mathrm{Zn}$ on the $(\mathrm{In}, \mathrm{Ga})$ site. The dimensionless figure of merit of $\mathrm{Cu}\left[\mathrm{In}_{1}\right.$ $\left.{ }_{\mathrm{x}} \mathrm{Ga}_{\mathrm{x}}\right]_{1-\mathrm{y}} \mathrm{Zn}_{\mathrm{y}} \mathrm{Te}_{2}(0 \leq \mathrm{x} \leq 1 ; 0 \leq \mathrm{y} \leq 0.15)$ is shown in panel $\left.\mathrm{c}\right)$. 
Figure 1

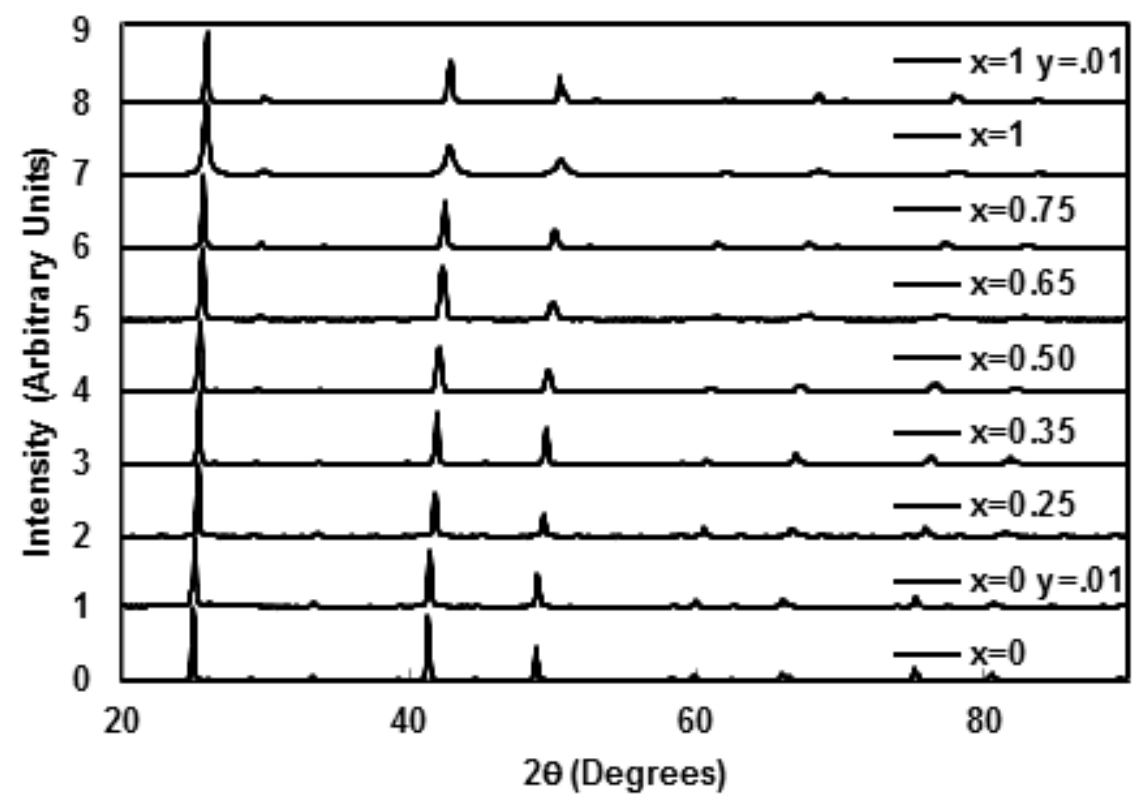


Figure 2a

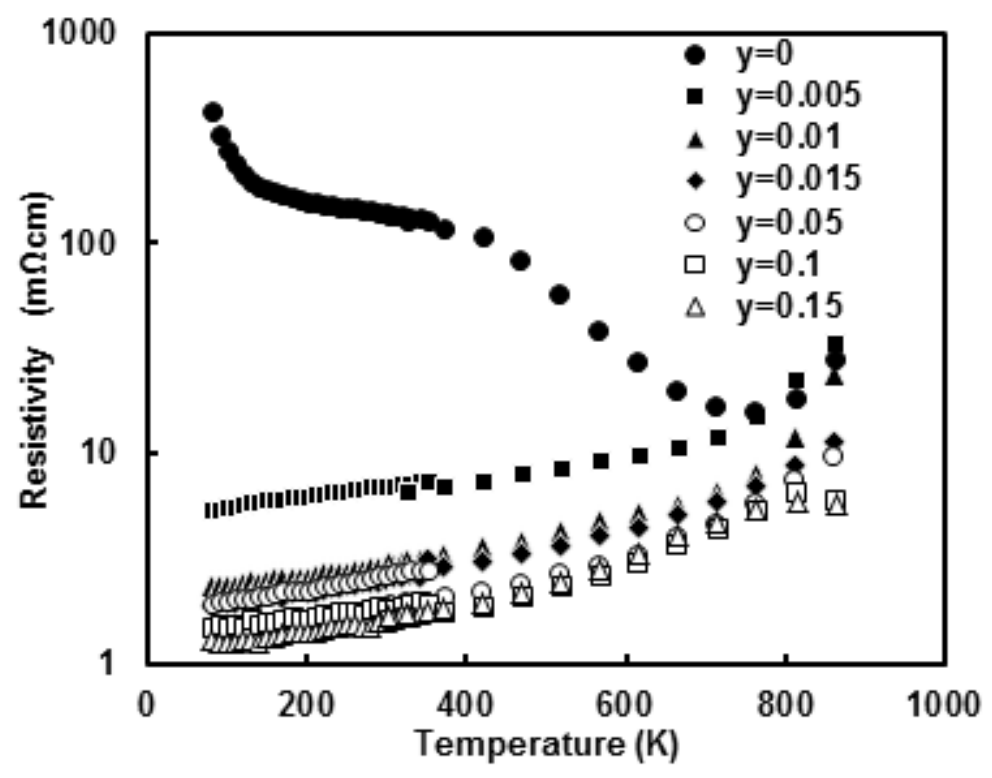

Figure 2b

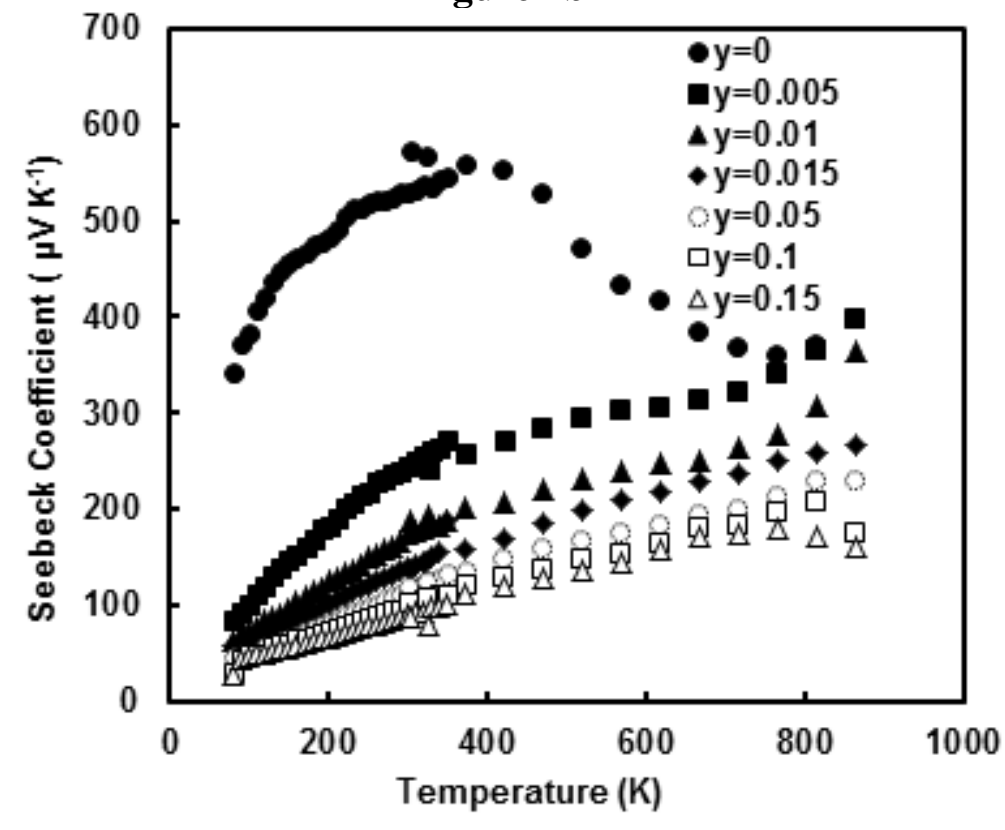


Figure 3a

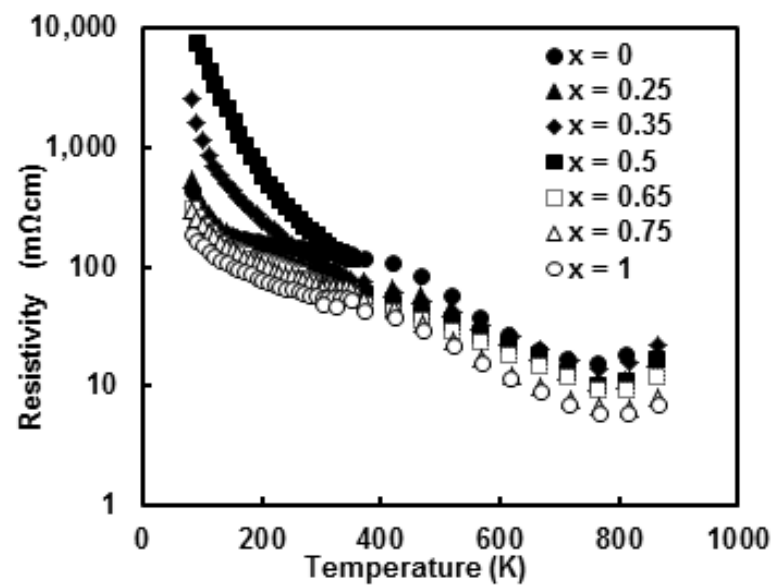

Figure 3b

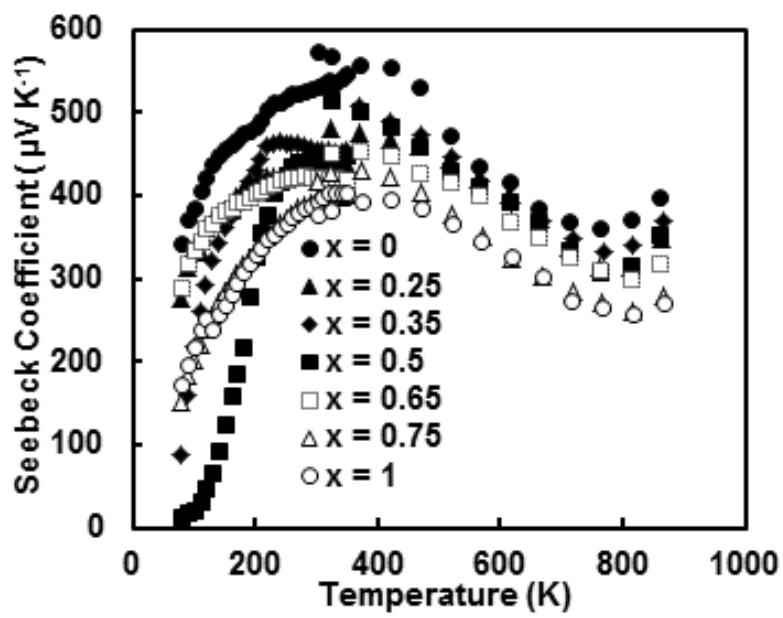

Figure 3c

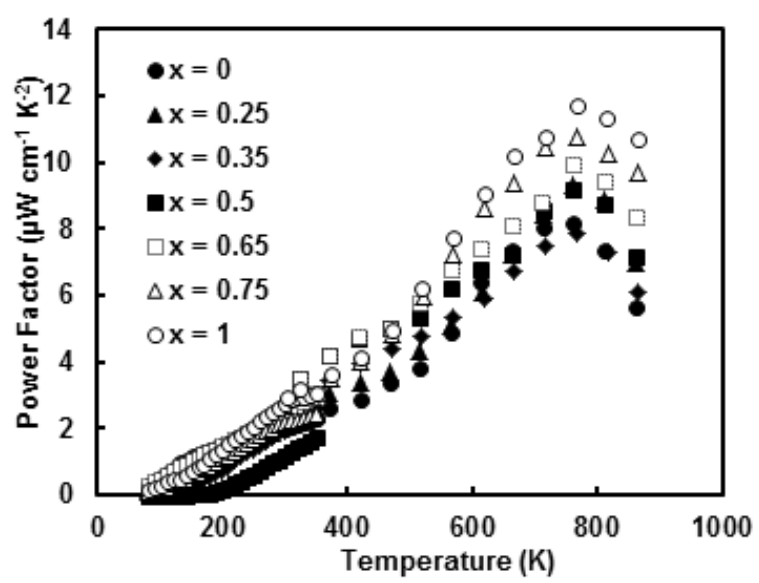


Figure 4a

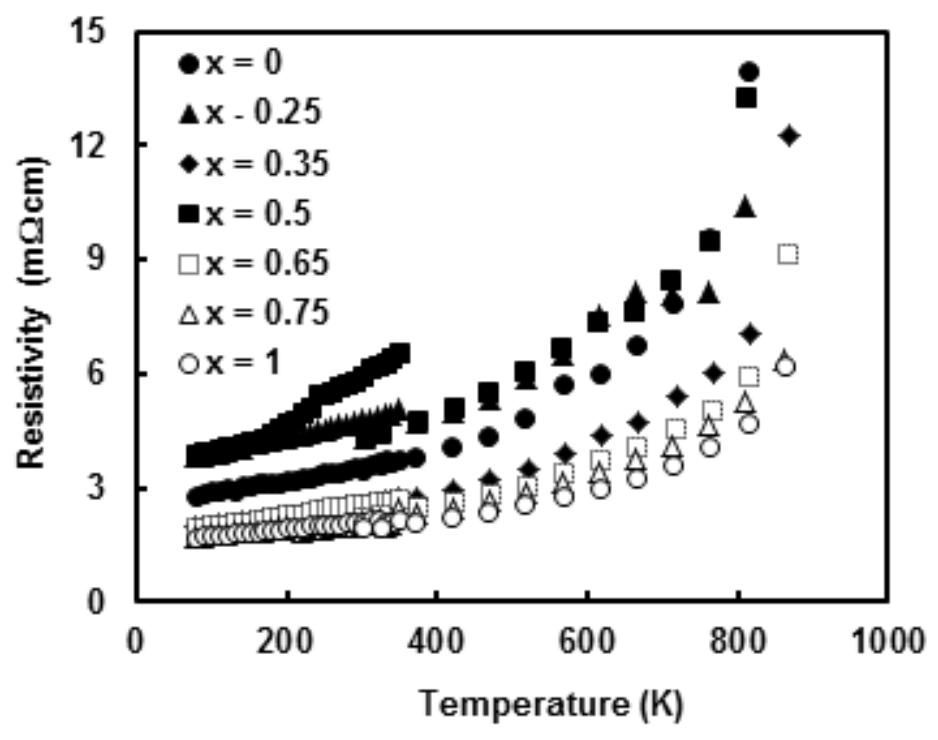

Figure 4b

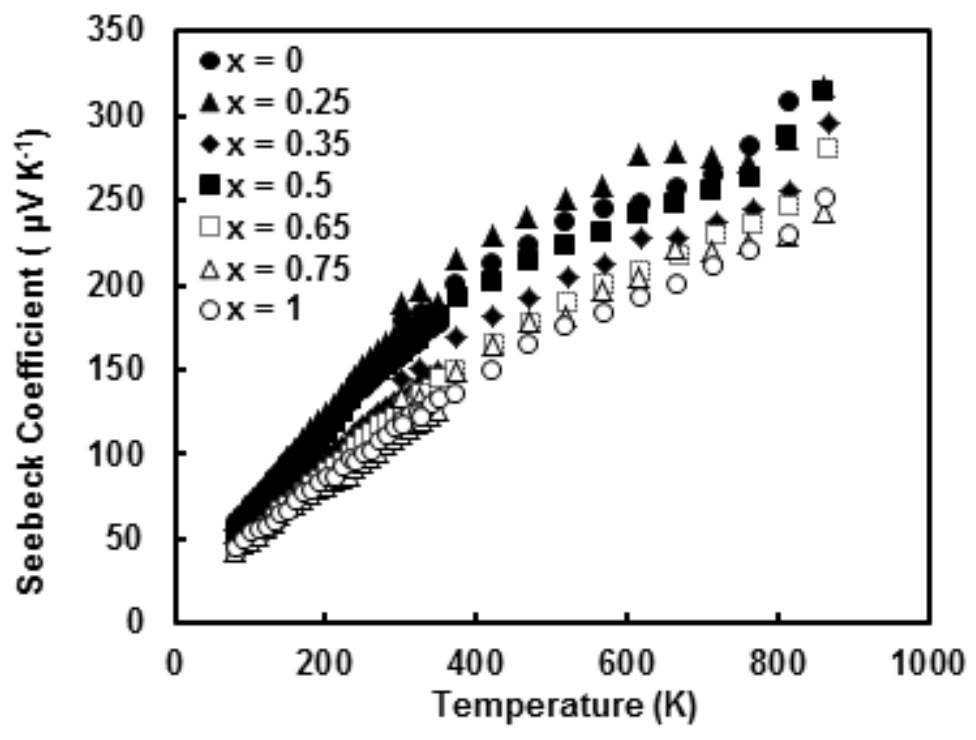


Figure 4c

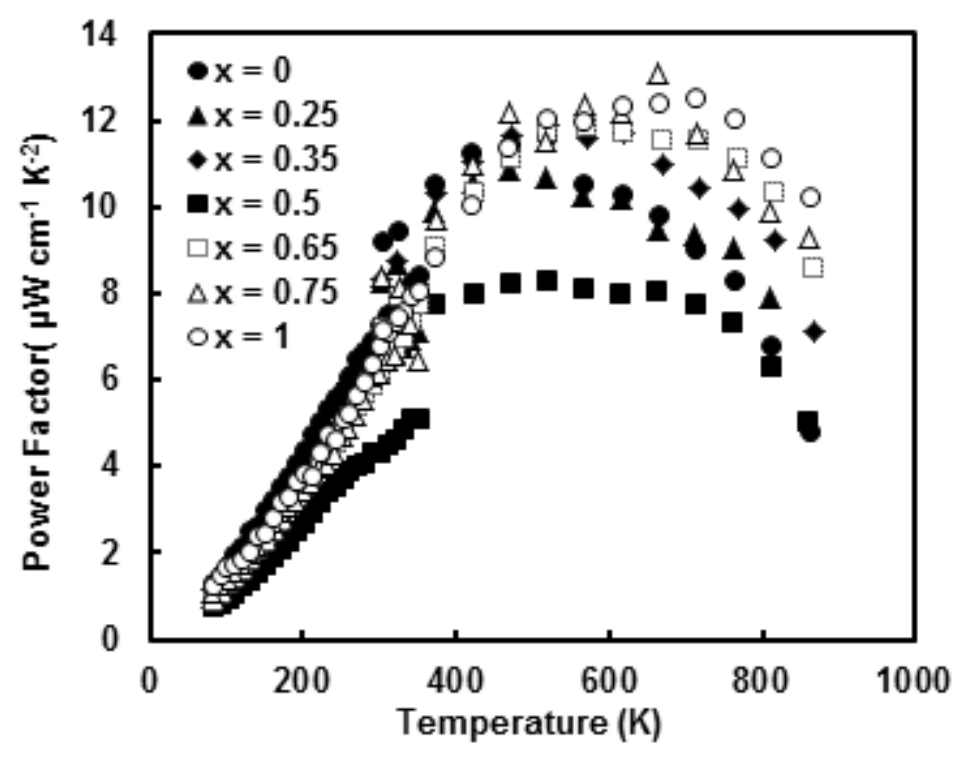


Figure 5a

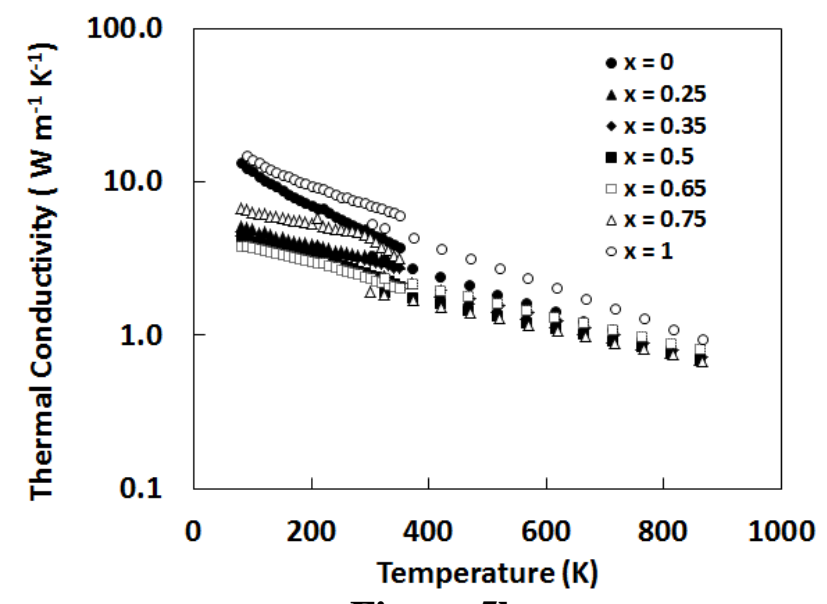

Figure 5b

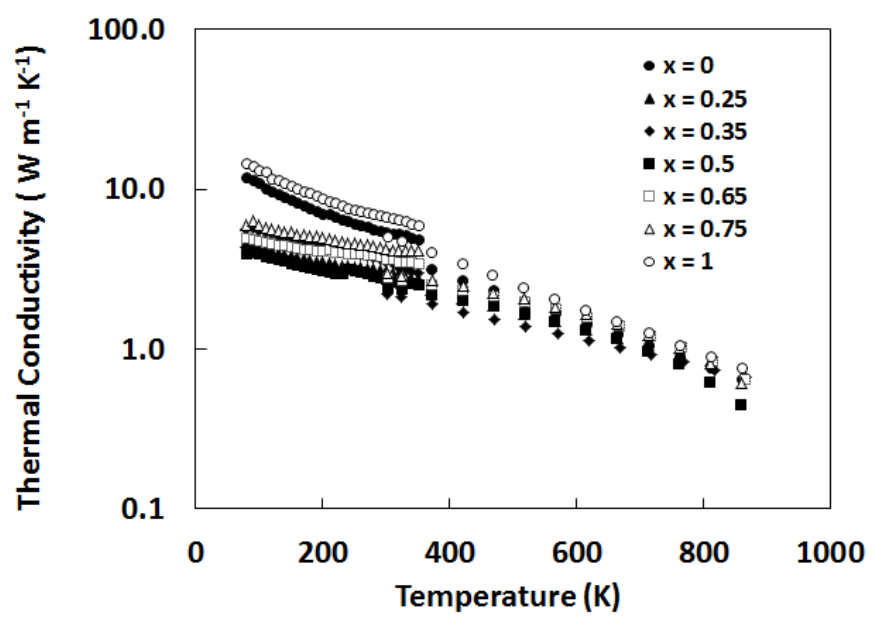

Figure 5c

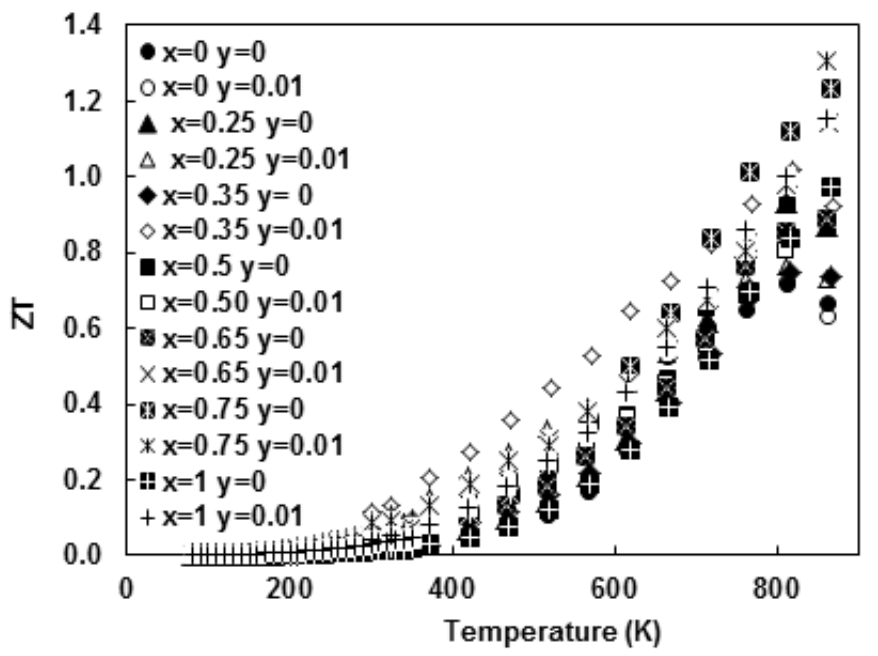

